\title{
Household interaction patterns and elderly persons caregivers' psychological wellbeing in Makueni sub-county, Kenya
}

\author{
Vincent M. Munywoki ${ }^{1 *}$, Sr. Dr. Elizabeth Ngozi Okpalaenwe ${ }^{2}$, \\ Dr. Urbanus Ndolo ${ }^{3}$
}

\section{ABSTRACT}

The psychosocial needs of the elderly are ever changing and this dynamism raises burden on caregivers of the elderly creating need for family support to balance their emotional disturbance. This study explored the relationship between household support systems and psychological wellbeing of elderly persons' caregivers in Makueni Sub-County, Makueni County, Kenya. The study adopted Bowen's Family Systems theory and used mixed method embedded design that combined correlation survey and phenomenology. The target population was caregivers, elderly persons and family members in 339 household families of elderly people receiving care at home. The sample size was 40 households in which 40 caregivers and family members each as well as 10 elderly were all sampled using purposive sampling. The 40 caregivers and family members were identified by virtue of the households while the elderly persons were identified on the basis of ability to respond to the questions. Data was collected using the Ryff's scale of psychological wellbeing (to measure wellbeing of caregivers) with reliability Cronbach's alpha of 0.86 and McMaster family device to assess the family interaction patterns of household family members with 0.7 coefficient. Data analysis was done using Statistical Package for Social Sciences (SPSS) - quantitative data was analyzed using univariate analyses and Pearson correlation. The findings of the study may be used in developing policies on care and protection of elderly people, but more importantly in enhancing counselling interventions for handling caregivers' psychological issues, hence facilitate family systems that support mental wellness of caregivers.

\section{Keywords: House Hold, Psychological Wellbeing, Family Functioning}

\begin{abstract}
A ccording to Kotkamp-Mothes, DanaSlawinsky, Hindermann, and Strauss (2005) in a study to review research on coping and psychological wellbeing in families of elderly cancer patients, findings therein highlighted the importance of the family as a source of social support. The findings too highlighted that family caregivers are second order patients to the family household as they often are the main part of the household to encounter psychological challenges associated with caregiving among the elderly. The
\end{abstract}

\footnotetext{
${ }^{1}$ Department of Psychology, the Catholic University of Eastern Africa, Kenya

${ }^{2}$ Psycho-Spiritual Institute, Marist International University College, Kenya

${ }^{3}$ Department of Sociology, the Catholic University of Eastern Africa, Africa

*Responding Author
}

Received: July 17, 2020; Revision Received: September 21, 2020; Accepted: September 23, 2020

(C) 2020, Munywoki V. M., Okpalaenwe E.N.\& Ndolo U.; licensee IJIP. This is an Open Access Research distributed under the terms of the Creative Commons Attribution License (www.creativecommons.org/licenses/by/2.0), which permits unrestricted use, distribution, and reproduction in any Medium, provided the original work is properly cited. 
researcher assumes this to imply that the caregiving role, can pose some challenges that are uniquely associated with caregiving on the caregiver, such that for instance, the pressure of meeting the dynamic needs of the elderly person, begets stress and other psychological disturbances related to the advancement of care. Hence, any attempt at mitigating challenges at the family level demand that the caregiver's wellbeing is considered too, primarily by seeking out ways of providing social and psychological support for the household as a whole and particularly the caregivers of elderly persons also considered in the intervention programmes. Part of the study's findings (Kotkamp-Mothes et al., 2005) highlighted that distress among family members is sometimes very similar to the distress experienced by cancer patients. Accordingly, the researcher presumes this to imply that interactions among the elderly person, the household members and the caregiver may often influence each other's psychological state. However, it is the interaction between the household members and caregivers that may have a higher influence on caregivers' welfare because of presumed expectations of caregiving by the household members on the caregiver being highly likely influential than the complaints of the elderly persons. In other words, interpersonal factors seem to have a specific impact on the adaptive capacities of elderly persons, their family members and the caregivers (Kotkamp-Mothes et. al., 2005).

It is the researchers' view that in line with the foregoing assertions, the description of caregivers as secondary patients indicates for the need to consider mechanisms to enhance the caregivers' wellbeing. For instance, caregivers may be predisposed to psychological conditions like psychosomatic disorders or even depression that they initially never had, and this condition will by extension affect their significant others. As such, attempts should be made towards identifying ways on how to protect family caregivers' health and safety can be enhanced and sustained (Hughes, 2008), because their caregiving demands place them at high risk for injury and adverse events. Besides, especially in African contexts, household based caregiving, family caregivers are more often than not volunteers towards the needs of the elderly and therefore undertake the duties as unpaid providers who often need help to learn how to become competent, safe volunteer workers through efforts by the household members to show moral and material support. This will further help the caregivers to be motivated towards caregiving as well as orient influence positively, the interactions with their significant others.

Furthermore, the family is not only the basic unit of society but also an important place for individual physical and psychological growth but as well affects the growth of every member and plays an important role in normal operation process of social system. According to a report on the Status and Implementation of National Policy on Aging in Kenya (Olum, 2015), majority of older people in Kenya are faced with host of problems that vary from economic, health, social and personal problems. The researcher presumes that more often than not, these challenges are ascribable to the social and economic circumstances of the household within which the elderly person's living arrangement. Further, the writer acknowledges the report's findings because the underscore the disconnect between what is perceived as the challenging issues for the elderly persons in terms of welfare and what exactly is seen as the main needs that the elderly persons desire. For instance, in the writer's view, high poverty levels, especially in most households in African contexts, have affected provision of basic financial assistance to the older persons. In particular, challenges of provision of food, shelter and health services have led to low level of livelihood as well as poor mental health outcomes of the elderly. As such, it can be 
argued that for example, when poverty affects the provision to care receiver, it is the expected that the interaction patterns of family towards support may too be influenced.

It has been established that care of dependent elderly relatives has a relationship with aging of populations and largely influence human resource in production, such that the burden of caring for the elderly can impair physical and mental health of caregivers (Rodriguez-Perez, Abreu-Sanchez, Rojas-Ocana, \& Rafael de-Pino-Casado, 2017). In other words, the impact on health and well-being of caregiver is attributed to stress associated with responsibility. Stress has potential of overwhelming and threatening psychological wellbeing and quality of life to caregivers of the elderly. Markey (2015) in a research on caregiving asserts that providing care for a relative is associated with psychological distress and a higher prevalence of psychological disorders - caregivers of elderly persons who are caring for a relative with a mental or physical illness may face challenges in relation to the task of caregiving thus influencing their level of burden. Thus, it is the researcher's view that in addition to the predisposing condition of elderly persons, other health related health conditions aggravate the needs of the elderly person in care. For instance, an elderly person with a pre-existing diabetic condition will incline the caregiver to provide additional care to manage the diabetic condition in addition to the normal caregiving needs.

Furthermore, according to a study by Senthil \& Kiran (2015) on family interaction pattern among Indian caregivers of elderly persons (patients) with epilepsy and alcohol dependence, using cross-sectional design, caregivers of patients with alcohol dependence were found to have poor family interaction with their significant others. Caregivers showed significant amount of problems in interactions and recommended for care of both patients and caregivers. However, in order to intervene effectively, an identification of poor interaction patterns among family members of caregivers imply that psychological wellness may be compromised, was an essential highlight in this study. The writer feels that this will facilitate for a proper assessment whether needs of the elderly provided with care and support at home had significant relationship with improvement of caregivers' psychological wellbeing using McMaster tool of family functioning rating scores. The writer adopts definition offered by Turliuc, Cludin and Robu (2016) who argued that family functioning refers to well-being and performance of family members in such domains as communication, cohesion, expressing affection, conflict resolution, problem solving, family task sharing, compliance with family rules or leadership (Beavers \& Hampson, 2003; Epstein et al., 2003). According to the writer, this definition of family functioning is comprehensive as it conceptualizes the various domains that influence family functioning.

Extensive research has been carried out on the physical and mental health effects of family caregiving (Shulz \& Sherwood, 2008) among informal caregivers. Further, a more definite association of caregiving has been offered by Shulz \& Sherwood (2008) who asserted that "Caregiving has all the features of a chronic stress experience: It creates physical and psychological strain over extended periods of time, is accompanied by high levels of unpredictability and uncontrollability, has the capacity to create secondary stress in multiple life domains such as work and family relationships, and frequently requires high levels of vigilance". The foregoing confirms the burdensome effect that caregiving can pose on caregiver's already dynamic life depending on the nature of relationship between the caregiver and elderly person. 
Moreover, Pinquart and Sorensen (2011) found that spouse caregivers reported higher levels of depressive symptoms, greater financial and physical burden, and lower levels of psychological well-being than adult child caregivers did. The researcher assumes that this aspect of emotional connection between caregiver and care recipient, influences psychological wellbeing as well as other physical provisions that influence affective aspects in the caregiver. However, in the researcher's view the foregoing circumstance only affirms that caregiver's burden is therefore a multi-dimensional phenomenon reflecting physical, psychological, social and financial consequences of caring for an elderly family member. The arguments on caregiving of impaired family members relate to caregiving of elderly people since both include participants who may not only have physical but also emotional burden from the family, and that it is these physical and emotional burdens that influence a caregiver's level of psychological wellbeing.

Existing research (Northhouse, Kafapodi, Schefenacker \& Weiss, 2012) has demonstrated that racial and ethnic differences exist within the level of psychological distress experienced by caregivers. While examining the psychological distress among the African communities, the study realized that the effects of stress on psychological domain in relation to quality of life is manifested in increased emotional distress, anxiety and depression; feelings of helplessness and loss of control; and difficulty in coping with caregiving roles. In other words, when the demand placed on caregivers exceed their resources, caregivers feel overwhelmed and report high stress levels.

According to Winefield et al (2012) in ascertaining the link between psychological wellbeing and psychological distress asserted that there is a long-standing acceptance that psychological distress (PD) in the form of anxiety, sadness, irritability, self-consciousness and emotional vulnerability is strongly correlated with physical morbidity, reduced quality and duration of life, and increased use of health services (Lahey, 2009). These assertions point out to the connectedness of the psychological wellbeing and psychological distress in determining caregivers' general health and by extension, their ability to offer the caregiving services to the optimum. In other words, in the event that the caregivers experience distress, then their caregiving role is affected negatively. According to the researcher, while agreeing with the foregoing assertions argues that this is perhaps the reason for which this study ought to be carried out to ascertain the connectedness of PW and PD among caregivers in order to advance a more Kenyan based framework for supporting distressed caregivers - and by so doing, influence caregiving to the elderly positively.

According to Pappin, M., Marais, L., Sharp C. Lenka M., Cloete J., Skinner D., and Serekoane Motsaathebe (2018) in a study carried out the Socio-economic Status and Socioemotional Health of Orphans in South Africa, linked socio-economic status (SES) and health status (both physical and mental). In the study it was established that a relationship existed between lower SES and higher incidence of mental health problems. The researcher presumes this to imply that caregivers who have lower socioeconomic status tend to experience a relatively higher likelihood of experiencing mental health challenges thus negatively affecting their psychological wellbeing. Thus, a caregiver who for example is from a poor background, may be expressly challenged in meeting the needs of the elderly persons in the process of caregiving that they experience psychological disturbances, thence affecting their psychological wellbeing negatively. 


\section{METHODOLOGY}

\section{Sample}

This study adopted mixed method approach. An embedded design using correlational and phenomenological study was used with an approach of two phases in data collection for both quantitative and qualitative research questions within the study (Hanson, 2005). The procedure offered a snapshot of the current state of affairs by seeking to establish a relationship between households' support systems and caregivers' wellbeing. The design was found appropriate for this study because it enabled the researcher to describe the circumstances of the caregivers and elderly people's household socioeconomic statuses as they were in Makueni Sub-county at the time of data collection and relate it to the levels of caregivers' wellbeing using their phenomenological expressions. Multistage sampling was used in preference to simple random sampling because the population was geographically diverse.

\section{Instruments}

Data was collected using the Ryff's scale of psychological wellbeing (to measure wellbeing of caregivers) with reliability Cronbach's alpha of 0.86 and McMaster family device to assess the family interaction patterns of household family members with 0.7 alpha.

\section{Procedure}

The Psychological Wellbeing of the elderly together with their family functioning were assed using the Psychological Wellbeing Scale and Family assessment Device respectively. Pearson correlation analysis was conducted to test relationship among variables.

\begin{tabular}{|c|c|c|c|c|c|c|c|}
\hline \\
\hline \multicolumn{8}{|c|}{ Table 1 Relationsh } \\
\hline & & $\begin{array}{l}\begin{array}{l}\text { Problem } \\
\text { solving }\end{array} \\
\text { soln }\end{array}$ & Communication & Roles & $\begin{array}{l}\text { Affective } \\
\text { respons. }\end{array}$ & $\begin{array}{l}\text { Affective } \\
\text { involvement }\end{array}$ & $\begin{array}{l}\text { General } \\
\text { functioning }\end{array}$ \\
\hline \multirow[t]{3}{*}{ Autonomy } & $\begin{array}{l}\text { Pearson } \\
\text { Correlation }\end{array}$ & .165 & -.113 & .257 & .063 & .292 & $-.125 \mathrm{r}$ \\
\hline & Sig. (2-tailed) & .309 & .488 & .110 & .698 & .068 & $.449 p$ \\
\hline & $\mathrm{N}$ & 40 & 40 & 40 & 40 & 40 & 39 \\
\hline \multirow[t]{3}{*}{$\begin{array}{l}\text { Environ. } \\
\text { mastery }\end{array}$} & $\begin{array}{l}\text { Pearson } \\
\text { Correlation }\end{array}$ & $.347^{*}$ & $.647^{* *}$ & $.500^{* *}$ & $.563^{* *}$ & .086 & $.543^{* *}$ \\
\hline & Sig. (2-tailed) & .028 & .000 & .001 & .000 & .598 & .000 \\
\hline & $\mathrm{N}$ & 40 & 40 & 40 & 40 & 40 & 39 \\
\hline \multirow[t]{2}{*}{$\begin{array}{l}\text { Personal } \\
\text { growth }\end{array}$} & $\begin{array}{l}\text { Pearson } \\
\text { Correlation }\end{array}$ & .295 & $.498^{* *}$ & $.390^{*}$ & $.348^{*}$ & .115 & $.485^{* *}$ \\
\hline & $\begin{array}{l}\text { Sig. (2-tailed) } \\
\mathrm{N}\end{array}$ & $\begin{array}{l}.065 \\
40\end{array}$ & $\begin{array}{l}.001 \\
40\end{array}$ & $\begin{array}{l}.013 \\
40\end{array}$ & $\begin{array}{l}.028 \\
40\end{array}$ & $\begin{array}{l}.479 \\
40\end{array}$ & $\begin{array}{l}.002 \\
39\end{array}$ \\
\hline \multirow[t]{2}{*}{$\begin{array}{l}\text { Positive } \\
\text { relations }\end{array}$} & $\begin{array}{l}\text { Pearson } \\
\text { Correlation }\end{array}$ & $.667^{* *}$ & $.698^{* *}$ & $.419^{* *}$ & $.365^{*}$ & .250 & $.646^{* *}$ \\
\hline & $\begin{array}{l}\text { Sig. (2-tailed) } \\
\mathrm{N}\end{array}$ & $\begin{array}{l}.000 \\
40\end{array}$ & $\begin{array}{l}.000 \\
40\end{array}$ & $\begin{array}{l}.007 \\
40\end{array}$ & $\begin{array}{l}.021 \\
40\end{array}$ & $\begin{array}{l}.120 \\
40\end{array}$ & $\begin{array}{l}.000 \\
39\end{array}$ \\
\hline \multirow[t]{2}{*}{$\begin{array}{l}\text { Purpose in } \\
\text { life }\end{array}$} & $\begin{array}{l}\text { Pearson } \\
\text { Correlation }\end{array}$ & $.376^{*}$ & $.592^{* *}$ & $.332^{*}$ & $.405^{* *}$ & .019 & $.461^{* *}$ \\
\hline & $\begin{array}{l}\text { Sig. (2-tailed) } \\
\mathrm{N}\end{array}$ & $\begin{array}{l}.017 \\
40\end{array}$ & $\begin{array}{l}.000 \\
40\end{array}$ & $\begin{array}{l}.036 \\
40\end{array}$ & $\begin{array}{l}.009 \\
40\end{array}$ & $\begin{array}{l}.909 \\
40\end{array}$ & $\begin{array}{l}.003 \\
39\end{array}$ \\
\hline \multirow[t]{2}{*}{$\begin{array}{l}\text { Self- } \\
\text { acceptance }\end{array}$} & $\begin{array}{l}\text { Pearson } \\
\text { Correlation }\end{array}$ & $.556^{* *}$ & $.725^{* *}$ & $.536^{* *}$ & $.536^{* *}$ & .267 & $.612^{* *}$ \\
\hline & $\begin{array}{l}\text { Sig. (2-tailed) } \\
\mathrm{N}\end{array}$ & $\begin{array}{l}.000 \\
40\end{array}$ & $\begin{array}{l}.000 \\
40\end{array}$ & $\begin{array}{l}.000 \\
40\end{array}$ & $\begin{array}{l}.000 \\
40\end{array}$ & $\begin{array}{l}.096 \\
40\end{array}$ & $\begin{array}{l}.000 \\
39\end{array}$ \\
\hline
\end{tabular}


Findings from the study indicated that correlation coefficient between autonomy and problem solving is (r: .165 p: .309), autonomy and roles is (r: .257 p: .110), autonomy and affective involvement is ( $\mathrm{r}: .292 \mathrm{p}$ : .068), autonomy and affective response is ( $\mathrm{r}: .063 \mathrm{p}$ : .698 ), autonomy and communication is (r: -.113 p: .488), and autonomy and general functioning is (r: $-.125 \mathrm{p}: .449$. According to this result, there is a low positive level relationship between autonomy and problem solving as well as roles, affective involvement and affective response; however, there is a negative and low level relationship between autonomy and communication and general functioning though there is insufficient evidence to conclude that there is a relationship between autonomy and family interaction patterns since the $p$ values are greater than .05 for all the family interaction patterns sub scales. The researcher's view that positive self-concept that boost autonomy may have had caregivers' enhanced ability in four out of six dimensions of psychological wellbeing. However, autonomy failure to indicate relationship with communication and general functioning dimensions could have been because of its negative effects on family pathology.

The results also indicated that correlation coefficient between environmental mastery and communication is (r: .647 p: .000), environmental mastery and affective response is ( $\mathrm{r}: .563$ p: .000), environmental mastery and general functioning is ( $\mathrm{r}: .543$ p: .000), environmental mastery and roles is (r: .500 p: .001), environmental mastery and problem solving is ( $\mathrm{r}: .347$ $\mathrm{p}$ : .028) and environmental mastery and affective involvement is (r: .086 p: .598). According to this result, there is a positive and medium level significant relationship between environmental mastery and communication, affective response, general functioning, roles and problem solving; however, indicate there is an insignificant positive and low-level relationship between environmental mastery and affective involvement.

Further it can be seen that, the correlation coefficient between personal growth and communication is ( $\mathrm{r}: .498 \mathrm{p}$ : .001), personal growth and general functioning is ( $\mathrm{r}: .485 \mathrm{p}$ : .002 ), personal growth and roles is (r: .390 p: .013), personal growth and affective response is (r: .348 p: .028), personal growth and problem solving is (r: .295 p: .065) and personal growth and affective involvement is (r: .115 p: .479). These findings prove that there is a positive and medium level significant relationship between personal growth and communication, general functioning, roles and affective response; however, there is a positive and low-level insignificant relationship between personal growth and problem solving and affective involvement.

In addition, it was found that correlation coefficient between positive relations and communication is ( $\mathrm{r}: .698 \mathrm{p}: .000$ ), positive relations and problem solving is ( $\mathrm{r}: .667 \mathrm{p}: .000$ ), positive relations and general functioning is ( $\mathrm{r}: .646 \mathrm{p}: .000)$, positive relations and roles is (r: .419 p: .007), positive relations and affective response is (r: .365 p: .021), and positive relations and affective involvement is (r: $.25 \mathrm{p}: .012$ ). According to this result, there is a positive and middle level significant relationship between positive relations and communication, problem solving, general functioning, roles and affective response; while there is a positive and low level insignificant relationship between positive relations and affective involvement.

From the results, it was revealed that correlation coefficient between purpose in life and communication is (r: .592 p:.000), purpose in life and general functioning is (r: .461 p: .003), purpose in life and affective response is (r: .405 p: .009), purpose in life and problem solving is ( $\mathrm{r}: .376 \mathrm{p}: .017)$, purpose in life and roles is ( $\mathrm{r}: .332 \mathrm{p}: .036)$ and purpose in life and 
affective involvement is ( $\mathrm{r}: .019 \mathrm{p}: .909)$. According to this finding, there is a significant positive and medium level relationship between purpose in life and communication, general functioning, affective response, problem solving and roles; however, there is an insignificant positive and very low level relationship between purpose in life and affective involvement.

Finally, analyzing the correlation coefficient the results are as follows: self-acceptance and communication is ( $\mathrm{r}: .725 \mathrm{p}$ : .000), self-acceptance and general functioning is ( $\mathrm{r}: .612 \mathrm{p}$ : .000 ), self-acceptance and problem solving is ( $\mathrm{r}: .056 \mathrm{p}: .000$ ), self-acceptance and roles is (r: .536 p: .000), self-acceptance and affective response is (r: .536 p: .000) and selfacceptance and affective involvement (r: .267 p: .096). These results show that there is a significant positive and strong relationship between self-acceptance and communication, general functioning, problem solving, roles and affective response; however, there is an insignificant positive and low level relationship between self-acceptance and affective involvement.

In the data analysis, participants showed desire of considering how to enhance themselves in each of the six areas of family functioning. Results of the analysis of each subscale indicated that participants positively identified with their level of family functioning with respect to problem-solving, communication, affective involvement, affective responsiveness, roles, behavior control, and general functioning. Paired t-tests were administered to detect statistically significant differences between the pre- and post-tests on the six subscales. The results indicated that a statistically significant change occurred at the .05 level on five out of the six dimensions. More specifically, caregivers noted positive results in their level of family functioning in the areas of problem-solving, communications, family roles, behaviour control, and the general functioning.

Findings of the study showed that families who were non-recipients of OPCT had better functioning interaction patterns than recipient families in all dimensions but indicated below average performance on affective involvement (mean== 2.8556; $\mathrm{SD}=.56488$ ). Their highest score was general functioning (mean=3.800; $\mathrm{SD}=1.21037)$. Households where elderly family member was benefiting from OPCT performed lowest on roles (mean=2.5273; $\mathrm{SD}=$ .72911 ) and affective involvement (mean= 2.5455; $\mathrm{SD}=.54574$ ). It was possible for the researcher to note that OPCT recipients were identified by government among vulnerable households with relatively poor socioeconomic status an important variable in the current study that was likely to have influenced functionality among family members on general interaction.

\section{DISCUSSION}

The study concluded that psychological well-being increases with increase in family pattern interactions. This is in agreement with findings of study on family functioning and its relationship to adolescent mental health done by Butler (2015) that found a significant amount of variance in life satisfaction and psychological wellbeing. The study reported life satisfaction and psychological wellbeing improve relationship between family functioning and externalizing behaviours.

According to the researcher, the findings show that on aggregate, interaction of family members that creates cohesiveness in each other's needs facilitates an enabling environment for caregivers' commitment in serving the elderly. However, the results indicate too that the respondents' comparatively lower rating of affective involvement meant that sometimes, 
individual needs and preferences for affection from others may reduce the caregivers' motivation for continued support of the elderly persons. This is true to the extent that positive feedback can easily enhance caregivers' levels of satisfaction and thereby directly increase one's experience of psychological wellbeing. For example, when a caregiver is appreciated by the household for the role that he/she plays in caring for the elderly, a sense of recognition is experienced and likely to make them be encouraged to continue performing the role. On the contrary, lack of appreciation, can bring about discouragement and as a consequence, reduce the level of commitment to the caregiving role among caregivers. In support to Thomas, Liu \& Umberson (2017) that relationships with family members are significant for wellbeing across the life course. This makes household relationships a key pillar in psychological wellbeing for caregivers.

\section{REFERENCES}

Butler, C. (2015). Family functioning and its relationship to adolescent mental health. (A doctoral research in Clinical Psychology, Royal Holloway, University of London.

Esptein, N. B., Balwin, L. M \& Bishop, D. S. (2003). The McMaster Family Assessment Device Journal of Marital and Family Therapy, 9 (2), 171-180.

Hanson, R. K. (2005). Case study research, design and methods. 3rd ed. Newbury Park: Sage

Kotkamp-Mothes, N., DanaSlawinsky, Hindermann, S. Strauss, B. (2005). Coping and psychological wellbeing in families of elderly cancer patients, Critical Reviews in Oncology/Hematology, Volume 55, Issue 3, September 2005, Pages 213-229

Markey, E. (2015). The Impact of Caregiving on the development of Major Depressive Disorder and Generalized Anxiety Disorder. Journal of European Psychology Students, 6(i), 17-24, DOI: http://dx.doi.org/ion 5334/jeps.cn

Northhouse, L.L., Kafapodi, M.C., Schefenacker \& Weiss D. (2012). The Impact of Caregiving on psychological well-being of Family Caregivers and Cancer Patients seminar in Oncology Nursing, Vol.28 No.4 pp236-245.

Olum, G.H. (2010). Report on status and implementation of national policy on aging in Kenya. Public Policy Institute (2015). Caregiving in the U.S. Comparisons among Population-Based Surveys. UNDESA.

Rodriguez-Perez, M., Abreu-Sanchez, A., Rojas-Ocana, M.J. \& Rafael de-Pino-Casado. (2017).

Current Directions in Psychological Science, Vol.4, NO.4 (Aug.1995), pp.99-104.Blackwall Publishing.

Ryff, C.D., (1995). Current Directions in Psychological Science, Vol.4, NO.4 (Aug.1995), pp.99-104. Blackwall Publishing.

Schulz, R. \& Sherwood, P. (2008). Physical and Mental Health Effects of Family Caregiving.

Senthil, M. \& Kiran, M. (2015). Family Interaction Patten among Caregivers of Patients with the socio-cultural context of family caregiving. The American Journal of nursing http://links.lww.com/A505

Thomas, P.A., Liu, H. \& Umberson, D. (2017). Family Relationships and Wellbeing. Gerontological Society of America. Innovation in Aging, Vol.1, No/3,1-11 doi:10.1093/geroni/igx 025

Winefield, H.R., Gill, T.K., Taylor, A.W. \& \& Rhiannon, M. P. (2012). Psychological wellbeing and psychological distress: is it necessary to measure both? Psychological Well-Being 2, 3 (2012). https://doi.org/10.1186/2211-1522-2- 
Household interaction patterns and elderly persons caregivers' psychological wellbeing in Makueni sub-county, Kenya

\section{Acknowledgments}

The author appreciates all those who participated in the study and helped to facilitate the research process.

\section{Conflict of Interest}

The author declared no conflict of interest.

How to cite this article: Munywoki V. M., Okpalaenwe E.N.\& Ndolo U. (2020). Household interaction patterns and elderly persons caregivers' psychological wellbeing in Makueni subcounty, Kenya. International Journal of Indian Psychology, 8(3), 1224-1232. DIP:18.01.128/20200803, DOI:10.25215/0803.128 